\title{
Yenidoğan Yoğun Bakım Ünitesinde Yatan Bebeğe Pozisyon
}

\section{Verme Uygulamaları}

\author{
Gamzegül ALTAY ${ }^{1}$
}

${ }^{1}$ Recep Tayyip Erdoğan Üniversitesi, Sağlık Bilimleri Fakültesi, Hemşirelik Bölümü, Rize, Türkiye, gamzegul.altay@erdogan.edu.tr (Sorumlu Yazar/Corresponding Author)

\begin{tabular}{|c|c|}
\hline Makale Bilgileri & ÖZ \\
\hline $\begin{array}{l}\text { Makale Geçmişi } \\
\text { Geliş: 25.01.2021 } \\
\text { Kabul: 08.04.2021 } \\
\text { Yayın: 25.08.2021 } \\
\text { Anahtar Kelimeler: } \\
\text { Yenidoğan, } \\
\text { Pozisyon, } \\
\text { YYBÜ, } \\
\text { Hemşirelik. }\end{array}$ & 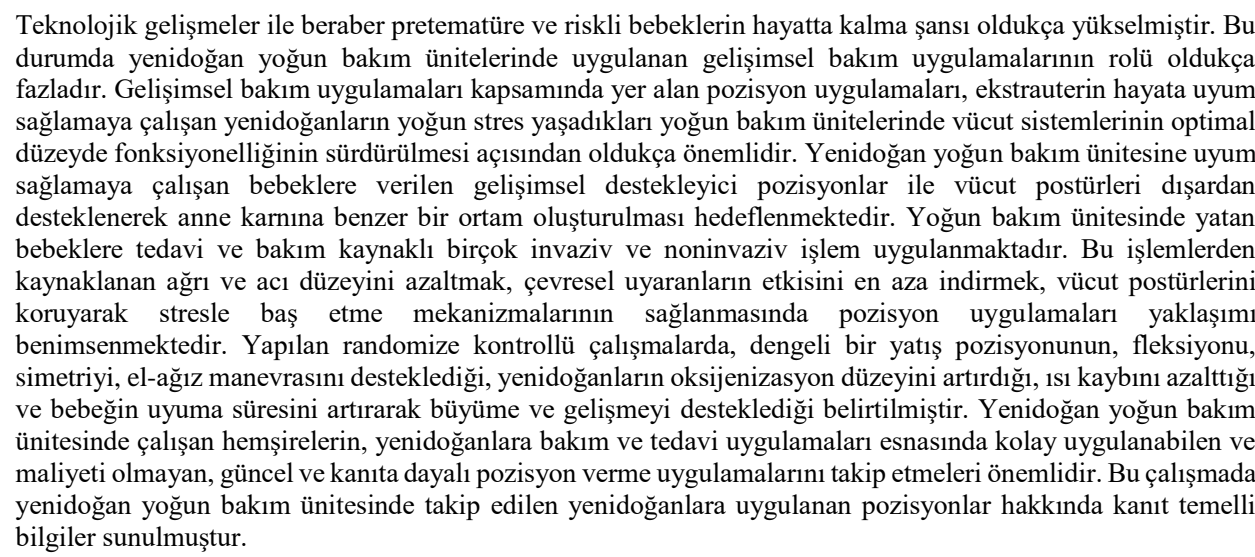 \\
\hline
\end{tabular}

\section{Positioning Practices of Neonates in Neonatal Intensive Care Unit}

\begin{tabular}{ll}
\hline Article Info & ABSTRACT \\
\hline Article History & Along with technological developments, the survival chance of pretermatur and risky neonates has increased \\
Received: 25.01.2021 & $\begin{array}{l}\text { considerably. In this case, the developmental care practices can apply a critical role in neonatal intensive care } \\
\text { units. Position practices, as one of the scopes of developmental care practices, are very important in terms of }\end{array}$ \\
Accepted: 08.04.2021 & maintaining the optimal functionality of body systems in intensive care units where newborns trying to adapt to \\
Published: 25.08.2021 & $\begin{array}{l}\text { extrauterine life experience with intense stress. It is aimed to create an environment similar to the mother's womb } \\
\text { by supporting the body postures with the developmental supportive positions given to neonate trying to adapt to }\end{array}$ \\
Keywords: & the neonatal intensive care unit. Many invasive and noninvasive procedures as treatment and care are applied to \\
Newborn, & neonate hospitalized in the intensive care unit. The position practice approach is used to reduce the level of \\
Position, & pain_caused by these procedures, minimize the effect of environmental stimuli, and develop their ability to cope \\
NICU, & with stress. A review of some randomized controlled studies, showed that a balanced lying flexion, symmetry, \\
Nursing. & and hand-mouth maneuver position, may increas the oxygenation level, decreas heat loss, and support growth \\
& and development by increasing the neonate's sleeping time. It is important for nurses to be up-to-date and familiar \\
& evidence-based positioning practices that are easy to apply without any cost during care practices in neonatal \\
intensive care unit. So, this article aims to present the, evidence-based information about the position practicing & as developmental care in neonatal intensive care unit.
\end{tabular}

*Bu derleme 26-29 Kasım 2020 tarihinde 1. Lisansüstü Hemşirelik Araştırmaları Sempozyum’unda sözel bildiri olarak sunulmuştur.

Atıf/Citation: Altay, G. (2021). Yenidoğan yoğun bakım ünitesinde yatan bebeğe pozisyon verme uygulamaları, Genel Sağllk Bilimleri Dergisi, 3(2), 143-151. 


\section{GíRIŞ}

Fetüs için normal yaşam ortamı anne karnıdır ve intrauterin ortam ile burada yer alan yapılar fetüsün büyüme-gelişmesinde önemli bir role sahiptir (Aydın ve Çiftçi, 2015). İntrauterin yaşam ortamlarından zamanından önce ayrılan prematüre bebekler, yenidoğan yoğun bakım ünitesi (YYBÜ) gibi oldukça farklı bir ortamla karşılaşırlar ve buna oryantasyon sağlamaya çalışırlar. Bu süreç içerisinde prematüre bebeklerin nörolojik ve fonksiyonel bozukluklarına yönelik davranış organizasyonunu sağlamada "Bireyselleştirilmiş Gelişimsel Bakım (BGB)" uygulamalarından yararlanılmakta ve çeşitli şekillerde uygulanmaktadır (Als ve McAnulty, 2011). BGB; iyileştirici çevre ve güvenli uykunun sağlanması, gelişimsel destekleyici aktivitelerden oluşan besleyici olmayan emme, pozisyon verme uygulamaları, kanguru bakımının verilmesi ve aile merkezli bakımın sağlanması aşamalarını içermektedir (Als ve McAnulty, 2011; Arpacı ve Altay, 2017; Coughlin vd., 2009; Sweeney vd., 2010).

Prematüre bebekler; vücut sistemlerinin immatür olması ve gelişimleri için uygun olmayan ekstrauterin yaşam ortamına ayak uydurmaya çalışmaları nedeniyle YYBÜ'ne alındıkları ilk zamanlarda yoğun stres yaşarlar (Aydın ve Yıldız, 2014; Çalışır ve Güler, 2017; Eras vd., 2013). Prematüre bebeklerin sinir sisteminin olgunlaşmasını desteklemeye yönelik uygulanan gelişimsel bakım uygulamaları ile stres yaratan uyaranların azaltılması hedeflenmektedir (Eras vd., 2013). Yenidoğanlar bu dönemde uygulanan gelişimsel bakım ile merkezi sinir sistemi ve diğer sistemlerinin istenilen düzeyde işlevselliğinin sürdürülmesi için özel pozisyonlarda yatırılmaya ihtiyaç duymaktadırlar (Als ve McAnulty, 2011; Hunter, 2010). YYBÜ'de pozisyon uygulamalarının temel amac1; bebeklerin tedavi ve bakım kaynaklı girişimlerinin sebep olduğu ağrl/acıdan ve çevreden kaynaklanan uyaranlardan en az düzeyde etkilenmelerini sağlamak, aynı zamanda postürlerini koruyarak stresle baş etme mekanizmalarını geliştirmektir (Hunter, 2010).

YYBÜ'nde ekstrauterin ortama uyum sağlamaya çalışan bebeklere gelişimsel pozisyon verme ile uterus içerisindeki gibi yer çekiminin olmadığı bir ortam oluşturularak yenidoğanların nöromotor sistemleri dışardan desteklenir (Hunter, 2010). Gelişimsel pozisyon verme uygulamaları ile büyüme gelişme süreci kolaylaşır, ağlama ve aşırı aktiviteler azaltılarak fonksiyonel aktiviteler için gerekli olan enerji korunur ve istenmeyen vücut postürünün oluşması önlenir (Als ve McAnulty, 2011; Hunter, 2004, 2010). Aynı zamanda bireyselleştirilmiş gelişimsel bakım aracılığıyla bebeğin kendini güvende hissetmesi sağlanarak bebeğin kendi kendini sakinleştirmesi ile fizyolojik açıdan stabilizasyon sağlanır (Aydın ve Çiftçi, 2015; Sathish vd., 2017). Bu çalışmada BGB uygulamaları kapsamında ele alınan pozisyon verme işleminin yenidoğan yoğun bakım ünitesinde kullanım şekilleri sunulmuştur.

\section{Bireyselleştirilmiş Gelişimsel Bakım ve Pozisyon Uygulamaları}

YYBÜ'nde gelişimsel destekleyici bakımın uygulamasında BGB uygulamalarının klinik açıdan birçok fayda sağladığı bilinmektedir (Taş Arslan ve Akkoyun, 2019). BGB faaliyetleri aracılığıyla ebeveynlerin memnuniyetlerinin arttığı, yenidoğanların fizyolojik parametrelerinin normal sınırlarda seyrettiği, hastanede kalış sürelerinin kısaldığı ve bakım maliyetlerinin azaldığı belirlenmiştir (Moddy vd., 2017). BGB uygulamaları kapsamında gelişimsel destekleyici aktiviteler içerisinde değerlendirilen "gelişimsel destekleyici pozisyon verme uygulamaları" bu kavramın temel bileşenleri arasında yer almaktadır (Arpacı ve Altay, 2017).

Doğru pozisyon sayesinde bebeklerin kendi kendini iyileştirmesine olanak sağlanmakta ve oluşabilecek nörolojik gelişim problemleri minimum seviyeye düşürülmeye çalış1lmaktadır. Gelişimsel pozisyon verme uygulamaları, bebeğin anne karnındaki fleksiyon postüründe, orta hatta, çevrelenmiş ve konforlu pozisyonunu koruyarak YYBÜ'deki bebeklerin nöromotor gelişimini desteklemeyi ve oluşabilecek problemleri azaltmayı amaçlamaktadır. Çevreleme ve fleksiyon pozisyonunun prematüre bebeklerde güvenli uykunun sağlanması ve duyu sistemlerinin gelişmesine katkıda bulunduğu 
belirtilmektedir (Hunter, 2010). Bebekler için uygun olmayan pozisyonlar ise özellikle prematürelerde kas-iskelet sistemi ile buna paralel gelişecek olan davranışsal bozukluklara, güvenli uykunun sağlanması ve sürdürülmesinde oluşabilecek sıkıntılara, kronik ağrıya ve uzun dönemde kalıcı postür bozukluklarına neden olmaktadır (Aydın ve Çiftçi, 2015; Madlinger-Lewis vd., 2014).

\section{Yanlış Pozisyonların Gelişimsel Etkileri}

Yenidoğan yoğun bakımda takip edilen yenidoğanlarda kullanılan tüm pozisyonlar (doğru- yanlış) nörodavranışsal organizasyon, kas iskelet sistemi gelişimi ve beslenme performansını etkilemektedir. Etkisiz pozisyon, kafatasında düzleşme veya değişik kafa şekillerinin ortaya çıkmasına yol açarken, prematüre bebekleri destekleyecek pozisyonların verilmemesinin fizyolojik stabilitenin azalmasına, bebeğin stres düzeyinin artmasına neden olacağı belirtilmektedir (Hunter, 2010).

Yanlış pozisyon uygulamalarına bağlı olarak kafatasının basınca maruz kalması ile deformiteler gelişir aynı zamanda sürekli maruz kalınan nazik basınçlar da kısmi deformitelere yol açabilir (Hunter, 2004, 2010). Yoğun bakım ünitelerinde yapılan tedavi girişimleri (ventilatör tedavisi nedeniyle endotrakeal tüp kullanımı ve yaptığı ağılık), bakım uygulamaları esnasında yapılan hatalardan (boyun yastıklarının sırta değil, boynun hemen altına yerleştirilmesi) kaynaklı boyun ve gövdede hiperekstansiyon ve buna bağlı sorunlar görülebilir. Üst ekstremitelerde yaygın olarak eksternal omuz rotasyonları ve skapular adduksiyonla beraber pozisyonel deformiteler görülmektedir (Hunter, 2004, 2010). Alt ekstremitelerde ise kurbağa pozisyonunda ( $M$ şeklinde) yatırılmaya bağlı olarak kalça abduksiyonu, eksternal rotasyon diz fleksiyonu, eksternal tibial torsiyon (tibia rotasyonu) ve diz eversiyonu gibi iskelet sistemine ait problemler sık karşılaşılan sorunlar arasında yer almaktadır (Hunter, 2004, 2010).

\section{Gelişimsel Destekleyici Pozisyon Uygulamaları}

BGB kavramlarından gelişimsel destekleyici pozisyon uygulamaları ile yenidoğanlara prone pozisyonu, yan yatış pozisyonu, supine ve cenin pozisyonu verilmektedir.

\section{Prone Pozisyonu}

Ekstrauterin çevrede yapılan düzenlemeler ile bebeğin kendini güvende hissetmesine olanak sağlayan prone pozisyonu, YYBÜ'lerinde tercih edilen en uygun yatış pozisyonudur (Hunter, 2004, 2010). Amerikan Pediatri Akademisi (AAP) yoğun bakım ünitesinde takip edilen prematüre bebekler için prone pozisyonunu önermektedir (Moon ve Fu, 2007). Bununla birlikte yapılan çalışmalarda, prone pozisyonu verilen bebeklerde Ani Bebek Ölümü Sendromu (ABÖS) vakalarının daha yüksek olduğu, prematüre bebeklerde ABÖS gelişme riskinin term bebeklere oranla daha yüksek olduğu belirtilmektedir (Goldwater, 2017; Picheansathian vd., 2009; Rohana vd., 2018).

Prone pozisyonunun yenidoğanlar üzerindeki faydaları fizyolojik açıdan incelendiğinde, bu bebeklerin oksijen ihtiyaçlarının daha az olduğu, solunum hızı ve apne periyodlarının azaldığı, kalp atım hızının düzenli olduğu ve beyne olan venöz dönüşün arttığı belirlenmiştir (Bembich vd., 2012; Shepherd vd., 2019). Prone pozisyonu verilen bebeklerin el-yüz ve el-ağız manevrası yaparak stresle daha kolay başa çıktıkları ve kendi kendilerini sakinleştirdikleri tespit edilmiştir (Shepherd vd., 2019).

Mörelius ve diğerlerinin (2016) yenidoğan yoğun bakımda tükürük kortizol seviyesini inceleyen 16 araştırmayı ele aldığı çalışmada, yenidoğanlara uygulanan invaziv girişimlerin (topuk kanı alma vs.) tükürük kortizol seviyesinde artışa neden olduğu ve yüzüstü pozisyon uygulamasının tükürük kortizol seviyesini düşürerek yenidoğanın stres düzeyini azalttığı belirlenmiştir (Mörelius vd., 2016).

Diğer pozisyonlara göre bu pozisyonda baş kontrolünü daha iyi sağlayan yenidoğanlarda, postür bozukluklarından kaynaklı asimetriler önlenerek, ekstremitelerin fleksiyon hareketinin kolaylaştı̆̆ ve ekstansiyonunun azaldığı saptanmıştır (Als ve McAnulty, 2011; Hunter, 2004; 2010). Bebeklerin bu 
pozisyonda uyuma periyodlarının artmasıyla beraber enerjilerini koruması sağlanarak kilo alımları arttı̆̆ 1 ve kalori harcanmalarının azaldığı görülmüştür. Aynı zamanda beslenme sonrası bebeklerin sessiz uyuma süresi artmış ve aktif ağlama süresi azalmıştır (Peng vd., 2014).

Prone pozisyonu yenidoğanların gastrik rezidü miktarını etkilemektedir (Yayan vd., 2018). Yayan ve diğerlerinin (2018) yürüttüğü çalışmada preterm bebeklere beslenme sonrası sırasıyla sağ lateral, sol lateral, supine ve prone pozisyonu verilmiş, gastrik kalıntıları nazogastrik tüp ile belirli aralıklarla değerlendirilmiştir. Beslenme sonrası sağ lateral ve prone pozisyonu verilen bebeklerin gastrik kalıntı miktarının diğer pozisyonlara göre daha az seviyede olduğu belirlenmiştir (Yayan vd., 2018).

Prone pozisyonunun bu olumlu etkilerine rağmen, bebeklerin motor gelişimini geciktirmesi ve acil tıbbi müdahale yapılmasını zorlaştırmasının yanı sıra çeşitli materyallerle desteklenmediği takdirde vücut yüzeyinde düzleşme, dolikosefali, lateral baş pozisyonu ve motor sisteme dair asimetriler geliştirmesi pozisyonun istenmeyen etkileri arasında yer almaktadır. Aynı zamanda bu pozisyonda yenidoğanların çevreyi gözlemi ve bakım verici ile yüz-yüze iletişimi zorlaşmaktadır (Aydın ve Çiftçi, 2015; Hunter, 2004, 2010).

Prone pozisyonu gövde ve kalçaya destek vererek bebek için daha iyi hale getirilebilir. Destek vermede sarılmış battaniye ve bebek bezi, bendy bummer, jel yastıklar, tek kullanımlık pamuklu ve polyester giysiler, köpük materyaller, içi dolu oyuncaklar sık kullanılan materyaller arasında yer almaktadır (Medlinger Lewis vd., 2014). Bu destek materyalleri başlıca ağırlık noktalarını olan omuz, gövde, pelvis gibi bölgelere konularak yenidoğanın akciğerlerinin rahat havalanması desteklenmektedir. Aynı zamanda gövde ve pelvisteki destekler ekstremitelere sağlanan yerçekiminin etkisini azaltarak, yenidoğanın fizyolojik postürünü kolaylaştırmaktadır (Gomes vd., 2019; Vergara ve Bigsby, 2004).

\section{Yan Yatış Pozisyonu}

Yan yatış pozisyonu, yer çekiminin ekstansör etkilerini azaltması, baş ile beraber el ve ayakların orta hat oryantasyonunu kolaylaştırması, aynı zamanda ekstremite aktivitelerini desteklemesi bakımından YYBÜ'de prone pozisyonunun kullanılamadığı durumlarda tercih edilen pozisyonlardan biridir (Aydın ve Çiftçi, 2015; Hunter, 2004, 2010). Fakat AAP, prone pozisyonu ile birlikte yan yatış pozisyonunun da ABÖS'na sebep olabileceğini vurgulamıştır (Moon ve Fu, 2007).

Yan yatışın hedefleri prone pozisyonu ile benzer olarak fleksiyonu kolaylaştırmak ve postür bozukluklarını önlemektir. Tek yönlü akciğer hastalığı olan bebeklerde etkilenen akciğer üst tarafta olacak şekilde yan yatış pozisyonu verilmesi, intertisiyel akciğer amfizemi olan bebeğe ise etkilenen akciğer alta gelecek şekilde yan yatış pozisyonu verilmesi ile daha iyi oksijenlenmenin sağlanacağı belirtilmiştir (Aydın ve Çiftçi, 2015; Hunter, 2004). Ayrıca sağ yan pozisyonda, sol yan ve supine pozisyonuna göre gastrik kalıntı miktarı daha az olduğu tespit edilmiştir (Yayan vd., 2018).

Aktif, irrite ve hipertonik ekstansiyon görülen bebeklere yan yatış pozisyonu verilerek fleksiyon pozisyonunun sürdürülmesi zor olmaktadır. Bu nedenle uygun materyal ile desteklenmeleri gerekmektedir. Desteklenmemiş yan yatış pozisyonundaki yenidoğanların karşılaştıkları gelişimsel sorunlar; omuz retraksiyonu, boyun ve gövdede hiperekstansiyon olarak açılanmıştır. Boyun hiperekstensiyonu yan yatış pozisyonunda uzun süre kalmış veya entübe yenidoğanlarda daha sık karşılaşılan bir durumdur (Hunter, 2010). Desteklenmemiş yan yatış pozisyonundan kaynaklı oluşabilecek problemleri önlemek, postüral dengeyi artırmak ve hiperekstansiyonu azaltmak için bazı yöntemler kullanılmaktadır. Üst ve alt ekstremiteler desteklenerek yan yatıştan supine veya prone pozisyonuna geçiş önlenmektedir. Bunun için yumuşak materyallerle sarmalama işlemi kullanılabilir. Bebek yatak kenarlarına sıkıştırılmış battaniye ile sarılarak denge arttırılabilir. Böylece fleksiyon pozisyonu sürdürülerek ve asimetrik pozisyonlar önlenebilir. Anne karnını taklit eden pozisyon materyalleri kullanılarak yenidoğanın pelvisi alttan ve arkadan desteklenir, bu sayede gerilme ve 
kaymalar en aza indirilerek yenidoğanın güvenliği sağlanır (Hunter, 2004, 2010).

\section{Supine Pozisyonu}

Supine pozisyonu, yenidoğanların anne karnındaki fleksiyon pozisyonunu desteklemediğinden YYBÜ’lerinde tercih edilen bir pozisyon değildir (Hunter, 2010). Buna rağmen ABÖS riskini azaltmak için bebeklerin supine pozisyonunda uyuması gerektiği önerisi bilimsel olarak kanıtlanmıştır (Sperhake vd., 2018). Supine pozisyonu hemşirelik bakım uygulamalarının kolaylıkla uygulanması ve bakım verici ile yüz-yüze iletişimi artırması açısından önemlidir. Özellikle neonatal dönemde abdomen veya gögüse uygulanan cerrahi girişimlerin stabilizasyonu evresinde sıklıkla kullanılan bir pozisyondur (Hunter, 2004). Supine pozisyonunun genel görevi yenidoğanın duyusal motor aktivitesini artırmak ve bebeğin gereksiz hareketlerden kaynaklanan enerji tüketimini önlemektir (Gomes vd., 2019; Vergara ve Bigsby, 2004).

Barsan Kaya ve diğerlerinin (2019) yürüttüğü çalışmada 37 haftadan küçük noninvaziv ventilasyon desteği alan 32 prematüre bebeğe supine ve prone pozisyonu verilerek solunum hızı, oksijen saturasyonu ve kan gazı analizleri karşılaştırılmıştır. Sonuç olarak noninvaziv solunum desteği alan preterm yenidoğanlarda supine pozisyonuna kıyasla prone pozisyonunun oksijenlenme düzeyinin daha iyi olduğu saptanmıştır (Barsan Kaya vd., 2019). Bu pozisyondaki bebeklerin gastrik rezidü miktarının diğer pozisyonlara göre daha fazla olduğu tespit edilmiştir (Yayan vd., 2018).

Gelişimsel yönden fleksiyondan daha çok ekstansiyonu destekleyen supine pozisyonunda, tekrar eden rastgele hareketler yuvalama ile azaltılmaktadır (Gomes vd., 2019; Vergara ve Bigsby, 2004). Battaniyeyle desteklenen supine-fleksör pozisyonunun entübe edilen bebeklerde kullanılması önerilmektedir (Çalışır ve Güven, 2017).

\section{Cenin Pozisyonu}

Cenin pozisyonu, "bebeği yuvaya alma yöntemi olarak da adlandırılmakla beraber, bebeğin kol ve bacaklarını el ile fleksiyonda tutularak, vücudu orta hatta yakın kapalı pozisyona alma işlemi” olarak tanımlanır. Bu yöntem uygulanırken bebeğe diğer gelişimsel pozisyonlar (lateral, supine ve prone) verilebilir (Apaydin Cirik ve Efe, 2020; Obeidat vd., 2009; Taplak ve Bayat, 2021). İntrauterin ortam desteği sağlayan bir pozisyondur (Ward-Larson vd., 2004). Bu pozisyon bebeğin kendini anne karnında hissetmesine ve kendi kendini sakinleştirmesine, ağrı ve stresle başa çıkma davranışlarını geliştirmesine, bebeklerin kendini güvende hissederek vücut kontrolünü sağlamasına yardımcı olur (Axelin vd., 2006; Hill vd., 2005; Obeidat vd., 2009; Taplak ve Bayat, 2021). Yenidoğanların motor gelişimini, büyüme ve gelişmesini destekleyen, uyku-uyanıklık örüntüsünü iyileştiren ve ağrının kontrol altına alınmasında olumlu etkileri olan bir yöntemdir (Apaydin Cirik ve Efe, 2020; Axelin vd., 2006; Hill vd., 2005; Perroteau vd., 2018).

Yapılan çalışmalarda, cenin pozisyonunun invaziv işlemler sırasında ağrıyı azaltmada etkili bir yöntem olduğu bildirilmektedir (Lopez vd., 2015; Küçükoğlu vd., 2015). Ayn1 zamanda bebeklerde 1s1 ve dokunsal uyarıyı sağlayarak, bebeklerin kendi düzenleyici sistemlerini aktive ettiği, bebeğin dikkatini aktif hale getirdiği, dış faktörlerden kaynaklı ağrllı uyaranları önlediği, endojen endorfin salınımına yol açtığı, spinal kordda yer alan ağrı uyaranlarının dağılımına yardımcı olduğu ve bebeğin hissettiği ağrıy önemli derecede azalttığı bildirilmektedir (Axelin vd., 2006; Hill vd., 2005).

Cenin pozisyonu verilen bebeklerin; uyku kalitesinin geliştiği, fizyolojik parametrelerinin daha kısa sürede stabil hale geldiği ve bebeğin pozisyona bağlı güven duygusu kazanmasına, motor gelişiminin desteklenmesine, aynı zamanda yenidoğanların enerjisini korumasına yardımcı olduğu belirtilmektedir (Perroteau vd., 2018). Bu pozisyon ile yenidoğanların sürekli aynı pozisyonda kalması engellenerek kas deformiteleri ve asimetrileri önlenir, gereksiz enerji harcaması azalarak bebeğin daha fazla dinlenmesi sağlanır ve güven duygusu geliştirerek yenidoğanın konforunu artırır (Kahraman vd., 
2018; Yıldırım Sarı ve Çiğdem, 2013).

\section{SONUÇ ve ÖNERILER}

Doğum sonrası anne yanı gibi güvenli bir ortamda olmak varken yenidoğan yoğun bakım ünitesinde bakım ve tedavi alan bebekler için uygun ortam şartlarının sağlanması son derece önemlidir. Özellikle prematüre bebekler immatür olan vücut sistemleri sebebiyle dışarıdan desteklenmeye ve uygun ortam şartlarına daha fazla ihtiyaç duyar ve YYBÜ'nde takip edilirler. Yenidoğanların YYBÜ'nde tedavi gördükleri süre boyunca gelişimini desteklemek için Bireyselleştirilmiş Gelişimsel Bakım Modeli geliştirilmiştir. Gelişimsel destekleyici pozisyon uygulamaları ile YYBÜ'nde bakım alan yenidoğanlara anne karnına benzer bir ortam oluşturmak esastır. Gelişimsel destekleyici pozisyon vermenin yenidoğanlarda kas-iskelet problemlerini önlemesi, tedavi ve bakım uygulamalarından kaynaklı ağrı ve stresi azaltması, güvenli uykunun sağlanması, konforu artırması ve bu sayede iyileşme sürecini hızlandırması gibi olumlu etkileri vardır. Gelişimsel destekleyici pozisyon uygulamaları ile özellikle preterm bebekler için anne karnı dışında güvenli bir ortam oluşturularak büyüme ve gelişme süreçleri desteklenir. Literatürde pozisyon uygulamaları ile ilgili randomize kontrollü çok sayıda çalışma mevcuttur. Bu çalışmalar hakkında sağlık profesyonelleri bilgilendirilerek, yenidoğanların bakımından birebir sorumlu olan yenidoğan hemşirelerinin pozisyon uygulamaları konusunda kanıta dayalı bilgi sahibi olması ve bunları uygulamalara aktarması büyük önem taşımaktadır.

\section{Finansal Destek}

Finansal destek alınmamıştır.

\section{Çıkar Çatışması}

Çıkar çatışması yoktur.

\section{Yazar Katkıları}

Tasarım/Design: G.A.; Veri toplama veya veri girişi yapma/Data collection or processing: G.A.;

Analiz ve yorum/Analysis or interpretation: G.A.; Literatür tarama/Literature search: G.A.;

Yazma/Writing: G.A.

\section{KAYNAKÇA}

Als, H., \& McAnulty, G. B. (2011). The newborn individualized developmental care and assessment program (NIDCAP) with kangaroo mother care: comprehensive care for preterm infants. Current Women's Health Reviews, 7(3), 288-301. https://doi.org/10.2174/157340411796355216.

Apaydin Cirik, V., \& Efe, E. (2020). The effect of expressed breast milk, swaddling and facilitated tucking methods in reducing the pain caused by orogastric tube insertion in preterm infants: A randomized controlled trial. International Journal of Nursing Studies, 104, 103532. https://doi.org/10.1016/j.ijnurstu.2020.103532

Arpacı, T., \& Altay, N. (2017). Yenidoğan Yoğun Bakım Ünitelerinde Bireyselleştirilmiş Gelişimsel Bakım: Güncel Yaklaşımlar. Türkiye Klinikleri Hemşirelik Bilimleri, 9(3), 245-254. https://doi.org/10.5336/nurses.2017-55289.

Aydın, D., \& Çiftçi, E. K. (2015). Neonatal Intensive Care Unit Nurses' Levels of Information Regarding Therapeutic Positions to be Applied to Preterm Newborns. The Journal of Current Pediatrics, 13, 21-30. doi:10.4274/jcp.26349.

Aydın, D., \& Yıldız, S. (2014). Effect of Turkish music that premature infants are made to listen during care on stress, oxygen saturation level and length of hospital stay. Journal of Human Sciences, 11(1), 1343-59.

Axelin, A., Salanterä, S., \& Lehtonen, L. (2006). 'Facilitated tucking by parents' in pain management of preterm infants-a randomized crossover trial. Early Human Development, 82(4), 241-247. https://doi.org/10.1016/j.earlhumdev.2005.09.012 .

Barsan Kaya, T., Aydemir, O., \& Tekin, A. N. (2019). Prone versus supine position for regional cerebral tissue 
oxygenation in preterm neonates receiving noninvasive ventilation. The Journal of Maternal-Fetal \& Neonatal Medicine, 1-6. https://doi.org/10.1080/14767058.2019.1678133.

Bembich, S., Oretti, C., Travan, L., Clarici, A., Massaccesi, S., \& Demarini, S. (2012). Effects of prone and supine position on cerebral blood flow in preterm infants. J Pediatr, 160(1), $162-164$. https://doi.org/10.1016/j.jpeds.2011.08.056.

Coughlin, M., Gibbins, S., \& Hoath, S. (2009). Core measures for developmentally supportive care in neonatal intensive care units: theory, precedence and practice. Journal of Advanced Nursing, 65(10), 2239-2248. https://doi.org/10.1111/j.1365-2648.2009.05052.x.

Çalışır, H., \& Güler, F. (2017). Yenidoğan Yoğun Bakım Ünitesinde Mekanik Ventilasyon Uygulanan Prematüre Bebeklere Pozisyon Verme. Türkiye Klinikleri Hemşirelik Bilimleri, 9(3), 227-232.

Eras, Z., Atay, G., Şakrucu, E. D., Bingöler, E. B., \& Dilmen, U. (2013). Yenidoğan yoğun bakım ünitesinde gelişimsel destek. Şişli Etfal Hastanesi Tıp Bülteni, 47(3), 97-103. 10.5350/SEMB2013470301

Goldwater, P. N. (2017). Sudden Infant Death Syndrome, Infection, Prone Sleep Position, and Vagal Neuroimmunology. Frontiers in Pediatrics, 5, 223. https://doi.org/10.3389/fped.2017.00223.

Gomes, E., Santos, C., Santos, A., Silva, A., França, M., Romanini, D. S., Mattos, M., Leal, A. F., \& Costa, D. (2019). Autonomic responses of premature newborns to body position and environmental noise in the neonatal intensive care unit. Respostas autonômicas de recém-nascidos prematuros ao posicionamento do corpo e ruídos ambientais na unidade de terapia intensiva neonatal. Revista Brasileira de terapia intensiva, 31(3), $296-302$. https://doi.org/10.5935/0103-507X.20190054.

Hill, S., Engle, S., Jorgensen, J., Kralik, A., \& Whitman, K. (2005). Effects of facilitated tucking during routine care of infants born preterm. Pediatric Physical Therapy, 17(2), 158-163. https://doi.org/10.1097/01.pep.0000163097.38957.ec.

Hunter, J. (2004). Positioning. Kenner C, McGrath J. (Ed.) Developmental Care of Newborns \& Infants. A Guide for Health Professionals. USA: Mosby (pp. 300-314).

Hunter J. (2010). Therapeutic positioning: neuro-motor development and beyond. In C. Kenner, J. M. McGrath, (Eds.), Developmental Care of Newborns \& İnfants: A Guide for Healthcare Professionals (2nd ed., pp. 285331). Chicago, IL: National Association of Neonatal Nurses.

Kahraman, A., Başbakkal, Z., Yalaz, M., \& Sözmen, E. Y. (2018). The effect of nesting positions on pain, stress and comfort during heel lance in premature infants. Pediatrics and Neonatology, 59(4), 352-359. https://doi.org/10.1016/j.pedneo.2017.11.010.

Küçükoğlu, S., Kurt, S., \& Aytekin, A. (2015). The effect of the facilitated tucking position in reducing vaccinationinduced pain in newborns. Italian Journal of Pediatrics, 41, 61. https://doi.org/10.1186/s13052-015-0168-9

Lopez, O., Subramanian, P., Rahmat, N., Theam, L. C., Chinna, K., \& Rosli, R. (2015). The effect of facilitated tucking on procedural pain control among premature babies. Journal of Clinical Nursing, 24(1-2), $183-191$. https://doi.org/10.1111/jocn.12657.

Madlinger-Lewis, L., Reynolds, L., Zarem, C., Crapnell, T., Inder, T., \& Pineda, R. (2014). The effects of alternative positioning on preterm infants in the neonatal intensive care unit: A randomized clinical trial. Research in Developmental Disabilities, 35(2), 490-497. 10.1016/j.ridd.2013.11.019

Moody, C., Callahan, T. J., Aldrich, H., Gance-Cleveland, B., \& Sables-Baus, S. (2017). Early Initiation of Newborn Individualized Developmental Care and Assessment Program (NIDCAP) Reduces Length of Stay: A Quality Improvement Project. Journal of Pediatric Nursing, 32, 59-63. https://doi.org/10.1016/j.pedn.2016.11.001.

Mörelius, E., He, H. G., \& Shorey, S. (2016). Salivary Cortisol Reactivity in Preterm Infants in Neonatal Intensive Care: An Integrative Review. International Journal of Environmental Research and Public Health, $13(3), 337$. https://doi.org/10.3390/ijerph13030337.

Obeidat, H., Kahalaf, I., Callister, L. C., \& Froelicher, E. S. (2009). Use of facilitated tucking for nonpharmacological pain management in preterm infants: a systematic review. The Journal of Perinatal \& Neonatal Nursing, 23(4), 372-377. https://doi.org/10.1097/JPN.0b013e3181bdcf77.

Peng, N. H., Chen, L. L., Li, T. C., Smith, M., Chang, Y. S., \& Huang, L. C. (2014). The effect of positioning on preterm infants' sleep-wake states and stress behaviours during exposure to environmental stressors. Journal of Child Health Care. 2014;18(4):314-325. https://doi.org/10.1177/1367493513496665.

Perroteau, A., Nanquette, M. C., Rousseau, A., Renolleau, S., Bérard, L., Mitanchez, D., \& Leblanc, J. (2018). Efficacy of facilitated tucking combined with non-nutritive sucking on very preterm infants' pain during the heel-stick procedure: A randomized controlled trial. International Journal of Nursing Studies, 86, $29-35$. https://doi.org/10.1016/j.ijnurstu.2018.06.007. 
Picheansathian, W., Woragidpoonpol, P., \& Baosoung, C. (2009). Positioning of Preterm Infants for Optimal Physiological Development: a systematic review. JBI Library of Systematic Reviews, 7(7), $224-259$. https://doi.org/10.11124/01938924-200907070-00001.

Rohana, J., Ishak, S., \& Wan Nurulhuda, W. (2018). Sudden infant death syndrome: Knowledge and practise in parents of preterm infants. Pediatrics International, 60(8), 710-713. https://doi.org/10.1111/ped.13605.

Sathish, Y., Edward Lewis, L., Angelitta Noronha, J., George, A., Snayak, B., Pai, M., Bhat, R., \& Purkayastha, J. (2017). Clinical outcomes of snuggle up position using positioning aids for preterm (27-32 weeks) infants. Iranian Journal of Neonatology. 8(1), 1-6. 10.22038/IJN.2016.7709 .

Shepherd, K.L., Yiallourou, S.R., Odoi, A., Brew, N., Yeomans, E., Willis, S., Horne, R., \& Wong, F. Y. (2019). Effects of Prone Sleeping on Cerebral Oxygenation in Preterm Infants. The Journal of Pediatrics, 204, 103110. https://doi.org/10.1016/j.jpeds.2018.08.076.

Sperhake, J., Jorch, G., \& Bajanowski, T. (2018). The prone sleeping position and SIDS. Historical aspects and possible pathomechanisms. International Journal of Legal Medicine, 132(1), 181- 185. https://doi.org/10.1007/s00414-017-1749-5.

Sweeney, J. K., Heriza, C. B., Blanchard, Y., \& Dusing, S. C. (2010). Neonatal physical therapy. Part II: Practice frameworks and evidence-based practice guidelines. Pediatric Physical Therapy, 22(1), 2-16. https://doi.org/10.1097/PEP.0b013e3181cdba43.

Taplak, A. Ş., \& Bayat, M. (2021). Comparison the Effect of Breast Milk Smell, White Noise and Facilitated Tucking Applied to Turkish Preterm Infants During Endotracheal Suctioning on Pain and Physiological Parameters. Journal of Pediatric Nursing, 56, e19-e26. https://doi.org/10.1016/j.pedn.2020.07.001

Taş Arslan, F., \& Akkoyun, S. (2019). Yenidoğan Yoğun Bakım Ünitesinde Gelişimsel Bakım Model ve Uygulamaları. Gümüşhane Üniversitesi Sağllk Bilimleri Dergisi, 8(4), $468-473$. https://dergipark.org.tr/tr/pub/gumussagbil/issue/50658/628284.

Moon, R. Y., \& Fu L. Y. (2007). Sudden Infant Death Syndrome. Pediatrics in Review, 28(6), $209-214$. https://doi.org/10.1542/pir.28-6-209.

Vergara, E. R., \& Bigsby, R. (2004). Elements of Neonatal Positioning. Paul H. Developmental and Therapeutic Interventions in The NICU. Baltimore, Brookes, p.177-203.

Yayan, E. H., Küçükoğlu, S., Dağ, Y. S., \& Karsavuran Boyraz, N. (2018). Does the Post-Feeding Position Affect Gastric Residue in Preterm Infants? Breastfeeding Medicine, 13(6), 438-443. https://doi.org/10.1089/bfm.2018.0028.

Yıldırım Sarı, H., \& Çiğdem, Z. (2013). Gestasyon haftalarına göre bebeğin gelişimsel bakımının planlanması. Dokuz Eylül Üniversitesi Hemşirelik Yüksekokulu Elektronik Dergisi, 6(1), 40-48. https://dergipark.org.tr/en/pub/deuhfed/issue/46815/587080.

Ward-Larson, C., Horn, R. A., \& Gosnell, F. (2004). The efficacy of facilitated tucking for relieving procedural pain of endotracheal suctioning in very low birthweight infants. MCN Am J Matern Child Nurs., 29(3), 151158. https://doi.org/10.1097/00005721-200405000-00004. 


\section{EXTENDED ABSTRACT}

With the technological developments, the survival chance of pretermatur and risky neonate has increased considerably. In this case, the developmental care practices can apply a critical role in neonatal intensive care units. After quitting the womb of a mother, which is a normal living environment for neonates, care and treatments are provided in complex units of neonatal intensive care unit. "Individualized Supportive Developmental Care" practices are used to provide a comprehensive care for neonates trying to adapt to this environment. The positional practices as one of the scope of these developmental care practices are performed to increase the comfort ofneonates during their care and treatment in neonatal intensive care units.

The purpose of position practicing in neonatal intensive care units are; to decrease pain caused by the treatment and care during interventions at a minimum level, minimize the stimuli originating from the environment, and develop their ability to cope with stress. Furthermore, by giving a developmental position to babies that are trying to adapt to the extrauterine environment, the neuromotor systems of the neonates are supported externally by creating an environment without gravity as in the uterus. In this way, the process of growth and development is facilitated, crying and excessive activities are reduced, the energy required for functional activities is preserved, and the formation of unwanted body posture is prevented. Also, the self-regulation conditions of the neonate are activated and the potential neurological development problems are minimized. Developmental positioning practices aim to support the neuromotor development of neonates in neonatal intensive care units and reduce the potential problems by preserving the baby's midline, surrounded, and comfortable position as in the womb. Unsuitable positions, especially in preterms, can cause behavioral disorders as well as physical disorders like musculoskeletal disorders, inability in maintaining safe sleep, long-term pain, and permanent posture disorders. Some examples of these disorders are external shoulder rotations and scapular adduction that are common in the upper extremities. In the lower extremities, hip abduction, external rotation, knee flexion, external tibial torsion (tibia rotation), and knee eversion due to lying in the frog position (M shape) are common.

Prone, supine, side-lying, and facilitated tucking are among the developmental supportive position practices. The prone position, which allows the baby to feel safe with the arrangements made in the extrauterine environment, is the most suitable lying position preferred in neonatal intensive care units.

The side-lying position is one of the positions preferred in neonatal intensive care units when the prone position cannot be used. The side-lying position reduces the extensor effects of gravity, facilitates the midline orientation of the hands, feet, and head, and also supports the extremity activities. In babies with active, irritable, and hypertonic extension, it is difficult to maintain the flexion position by giving the side-lying position. Therefore, they must be supported with suitable material. Using position materials to imitate the mother's womb environment, the pelvis of the neonate is supported from below and behind. In this way, stress and slippage are minimized.

The supine position is not a preferred position in neonatal intensive care units as it does not support the flexion position of the neonate as in the womb. The general task of the supine position is to increase the sensorymotor activity of the neonate and prevent the energy consumption from unnecessary movements. In the supine position, supports extension rather than flexion, however, in a developmental position, repetitive random movements are reduced by nesting.

Facilitated tucking is also called "the method of taking the baby into the nest, is defined as the process of placing the body close to the midline by holding the baby's arms and legs in hand flexion". While applying this position, other developmental positions like lateral, supine, and prone can be given to the baby.

In facilitated tucking; it is stated that sleep quality improves, physiological parameters can become stable in a shorter time, as well as it can it helps the baby to gain a sense of confidence depending on the position, support motor development, and protect the energy of neonate. With this position, newborns are prevented from staying in the same position continuously, preventing muscle deformities and asymmetries, reducing unnecessary energy expenditure, allowing the baby to rest more, and increasing comfort by developing a sense of confidence.

So, it is extremely important to provide appropriate environmental conditions for neonates who receive care and treatment in the neonatal intensive care unit, and reducing their level of pain caused by invasive and noninvasive procedures and increasing their comfort. As the evidence showed these environmental conditions can be achieved with position practices, which is one of the scopes of individualized developmental care. Therefore, the quality of care increases in neonatal intensive care units. So, it is recommended that nurses and other health professionals who are responsible for the care of neonate research on different position practices and applied their results in practice. 